\title{
THE STOKES SYSTEM IN THE INCOMPRESSIBLE CASE-REVISITED
}

\author{
RAINER PICARD \\ Institut für Analysis, FR Mathematik, TU Dresden \\ D-01069 Dresden, Germany \\ E-mail: rainer.picard@tu-dresden.de
}

\begin{abstract}
The classical Stokes system is reconsidered and reformulated in a functional analytical setting allowing for low regularity of the data and the boundary. In fact the underlying domain can be any non-empty open subset $\Omega$ of $\mathbb{R}^{3}$. A suitable solution concept and a corresponding solution theory is developed.
\end{abstract}

0. Introduction. It may seem somewhat surprising that even for the linearized incompressible Navier-Stokes equations there appears to be no "soft analysis" solution theory as is available e.g. for evolutionary equations. This is partly due to the fact that this system is what is known as a partial differential-algebraic equation (PDAE). In this note we want to analyze the Stokes problem, which is just the leading part of the linearized incompressible Navier-Stokes equations, with functional analytical means in order to develop a solution concept that avoids unnecessary regularity assumptions on data and quality of the boundary. The approach is based on concepts developed in $[2,3]$.

The Stokes system can be given in the form

$$
\begin{array}{r}
\operatorname{div} v=0, \\
\partial_{0} v-\operatorname{Div} T=f,
\end{array}
$$

where the system is completed by the material relation

$$
T=C \operatorname{Grad} v-p 1_{3 \times 3} .
$$

2000 Mathematics Subject Classification: Primary 35Q30, 76D07.

Key words and phrases: incompressible Navier-Stokes equations, Stokes problem, Stokes initial value problem, Stokes operator, Sobolev lattices, PDAE.

The paper is in final form and no version of it will be published elsewhere. 
Here the so-called viscosity tensor $C$ commonly characterizes an isotropic medium, i.e.

$$
C=\left(\begin{array}{cccccc}
2 \mu+\kappa & \kappa & \kappa & 0 & 0 & 0 \\
\kappa & 2 \mu+\kappa & \kappa & 0 & 0 & 0 \\
\kappa & \kappa & 2 \mu+\kappa & 0 & 0 & 0 \\
0 & 0 & 0 & \mu & 0 & 0 \\
0 & 0 & 0 & 0 & \mu & 0 \\
0 & 0 & 0 & 0 & 0 & \mu
\end{array}\right), \quad \mu, \kappa \in \mathbb{R}
$$

when-following Voigt's notation - symmetric matrices $S=\left(S_{i, j}\right)_{i, j=1,2,3}$ are represented as 6-component vectors

$$
S=\left(\begin{array}{c}
S_{11} \\
S_{22} \\
S_{33} \\
S_{23} \\
S_{31} \\
S_{12}
\end{array}\right)
$$

Note that the $3 \times 3$ identity matrix is in this notation identified with the vector

$$
\left(\begin{array}{l}
1 \\
1 \\
1 \\
0 \\
0 \\
0
\end{array}\right)
$$

The formal differential operators Grad and Div can then be interpreted (see e.g. [1], chapter 11) as

$$
\operatorname{Grad}:=\left(\begin{array}{ccc}
\partial_{1} & 0 & 0 \\
0 & \partial_{2} & 0 \\
0 & 0 & \partial_{3} \\
0 & \partial_{3} & \partial_{2} \\
\partial_{3} & 0 & \partial_{1} \\
\partial_{2} & \partial_{1} & 0
\end{array}\right)
$$


and its formal transpose (or $L^{2}$-skew-adjoint)

$$
\operatorname{Div}:=\left(\begin{array}{cccccc}
\partial_{1} & 0 & 0 & 0 & \partial_{3} & \partial_{2} \\
0 & \partial_{2} & 0 & \partial_{3} & 0 & \partial_{1} \\
0 & 0 & \partial_{3} & \partial_{2} & \partial_{1} & 0
\end{array}\right)
$$

One finds that the eigenvalues of $C$ are $\mu, 2 \mu+3 \kappa, 2 \mu$ with multiplicities 3,2 and 1 , respectively. The quantities $\mu, \kappa$ are known as Lamé constants and for the medium we must have

or

$$
\begin{aligned}
& \mu>0,2 \mu+3 \kappa>0 \\
& \mu>0,0<\kappa+\frac{2}{3} \mu
\end{aligned}
$$

in order to ensure that $C$ is positive definite. We mention this only for motivational reasons. In our later development it will turn out that $C$ can be fairly arbitrary, thus allowing for inhomogeneous and even anisotropic media. Considering the above system of equations, which formally constitutes a PDAE, i.e. a partial differential algebraic equation, amounts to studying the system matrix

$$
\left(\begin{array}{ccc}
0 & \operatorname{div} & 0 \\
0 & \partial_{0} & -\operatorname{Div} \\
(\cdot) 1_{3 \times 3} & -C \operatorname{Grad} & 1
\end{array}\right)
$$

The resulting system matrix can be transformed into a more "symmetric" form. Starting with the matrix trace operation given by $\mathbb{C}^{3 \times 3} \rightarrow \mathbb{C}, T \mapsto \operatorname{trace}(T)$, we obtain as trace* the operator $\mathbb{C} \rightarrow \mathbb{C}^{3 \times 3} x \mapsto x 1_{3 \times 3}$, and we get

$$
\operatorname{trace} C^{-1} T=\operatorname{trace}\left(\operatorname{Grad} v-p C^{-1} 1_{3 \times 3}\right)=\operatorname{trace} \operatorname{Grad} v-\operatorname{trace} C^{-1} \operatorname{trace}^{*} p .
$$

Since trace $\operatorname{Grad} v=\operatorname{div} v$, we have

$$
\operatorname{trace} C^{-1} T=\operatorname{div} v-\operatorname{trace} C^{-1} \operatorname{trace}^{*} p
$$

and we obtain the formally equivalent system matrix *)

$$
\left(\begin{array}{ccc}
\operatorname{trace} C^{-1} \text { trace* }^{*} & 0 & \operatorname{trace} C^{-1} \\
0 & \partial_{0} & -\operatorname{div} \\
\text { trace* }^{*} & -C \text { Grad } & 1
\end{array}\right)
$$

$\left.{ }^{0 *}\right)$ In the isotropic case we find

$$
\begin{aligned}
\frac{1}{2 \mu+\kappa} \operatorname{trace}(C S) & =\frac{1}{2 \mu+\kappa} \sum_{i} \sum_{k, l} C_{i i k l} S_{k l}=\frac{1}{2 \mu+\kappa} \sum_{i, k} C_{i i k k} S_{k k} \\
& =\frac{2 \mu}{2 \mu+\kappa} \sum_{k} S_{k k}+\frac{\kappa}{2 \mu+\kappa} \sum_{k} S_{k k}=\operatorname{trace}(S)
\end{aligned}
$$

for all symmetric $S$. With $S=C^{-1} T$ we have trace $C^{-1} T=\frac{1}{2 \mu+\kappa}$ trace $T$. Thus in particular,

$$
\text { trace } C^{-1} \operatorname{trace}^{*} p=\frac{1}{2 \mu+\kappa} \text { trace } \operatorname{trace}^{*} p=\frac{3}{2 \mu+\kappa} p .
$$


where

$$
\operatorname{trace}^{*}=1_{3 \times 3}=\left(\begin{array}{c}
1 \\
1 \\
1 \\
0 \\
0 \\
0
\end{array}\right)
$$

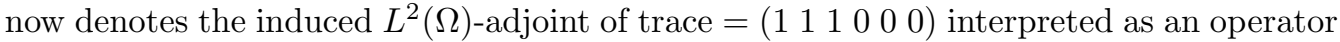
from $\left(L^{2}(\Omega)\right)^{6}$ to $L^{2}(\Omega)$. Here $\Omega$ is a non-empty open set in $\mathbb{R}^{3}$ occupied by a medium described by the tensor $C$. It seems natural to consider this system in a weighted $L^{2}(\Omega)$ space denoted by $\mathcal{H}_{0}$ with inner product of the form

$$
\langle\cdot \mid \cdot\rangle_{0}+\langle\cdot \mid \cdot\rangle_{0}+\left\langle\cdot \mid C^{-1} \cdot\right\rangle_{0} \cdot
$$

The notation $\langle\cdot \mid \cdot\rangle_{0}$ refers to the standard (one-, three- or six-component) $L^{2}(\Omega)$-innerproduct. In other words, we have

$$
\mathcal{H}_{0}=L^{2}(\Omega) \oplus L^{2}(\Omega) \oplus C^{1 / 2} L^{2}(\Omega) .
$$

The weight $C$ can be chosen completely general, in particular we may have anisotropies and inhomogeneities in the medium. The only requirement is that $C$ is a positive definite, bounded selfadjoint mapping in the space of symmetric matrices with $L^{2}(\Omega)$-entries denoted here simply as $L^{2}(\Omega)$ again. This weight is chosen such that Div is formal adjoint of $-C$ Grad. Thus,

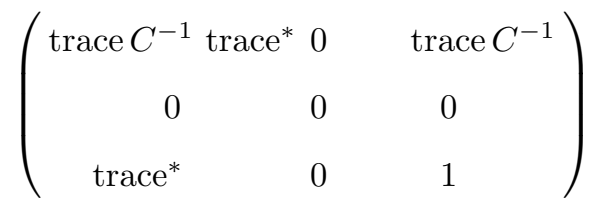

is a selfadjoint bounded operator in $\mathcal{H}_{0}$ and we are led to discuss the system matrix

$$
\left(\begin{array}{ccc}
\operatorname{trace} C^{-1} \text { trace* }^{*} & 0 & \operatorname{trace} C^{-1} \\
0 & \partial_{0} & -\mathrm{Div} \\
\text { trace* }^{*} & -C \text { Grad } & 1
\end{array}\right)
$$

as acting in the tensor product space $L^{2}\left(\mathbb{R}, \mu_{\eta}\right) \otimes \mathcal{H}_{0}$ for sufficiently large $\eta \in \mathbb{R}_{>0}$, where $L^{2}\left(\mathbb{R}, \mu_{\eta}\right)$ denotes the space of (equivalence classes of) complex-valued, square-integrable functions over $\mathbb{R}$ (with respect to almost everywhere equality as equivalence relation) equipped with the weighted measure

$$
\mu_{\eta}(M):=\int_{M} \exp (-2 \eta t) d t, \quad \eta \in \mathbb{R}_{>0} .
$$

Its Hilbert space norm will be denoted by $|\cdot|_{\eta, 0}$. Defining $\partial_{\eta}:=\mathrm{i} \mathfrak{I m} \partial_{0}$, where $\partial_{0}$ denotes the normal realization of the differentiation operator in $L^{2}\left(\mathbb{R}, \mu_{\eta}\right)$. Note that $\mathfrak{R e} \partial_{0}=\eta$, 
for details of the framework see $[3,2]$. We recall, in particular, that the domain $D\left(\partial_{0}^{k}\right)$ equipped with the norm $\phi \mapsto\left|\partial_{0}^{k} \phi\right|_{\eta, 0}$ is again a Hilbert space denoted by $H_{k}\left(\partial_{\eta}+\eta\right)$, $k \in \mathbb{N}$. The dual spaces are denoted by $H_{-k}\left(\partial_{\eta}+\eta\right)$ for $k \in \mathbb{N}$. In any case, $H_{k}\left(\partial_{\eta}+\eta\right)$ can be understood as the completion of $D\left(\partial_{0}^{|k|}\right)$ with respect to the norm $\phi \mapsto\left|\partial_{0}^{k} \phi\right|_{\eta, 0}$, $k \in \mathbb{Z}$. By construction $\partial_{0}:=\partial_{\eta}+\eta$ maps $H_{k+1}\left(\partial_{\eta}+\eta\right)$ unitarily onto $H_{k}\left(\partial_{\eta}+\eta\right)$ for every $k \in \mathbb{Z}$.

In the boundary value case we consider the revised incompressible Stokes system in the Dirichlet boundary condition case

$$
\left(\begin{array}{ccc}
\operatorname{trace} C^{-1} \operatorname{trace}^{*} & 0 & \operatorname{trace} C^{-1} \\
0 & \partial_{0} & - \text { Div } \\
\text { trace* }^{\circ} & -C \overbrace{\text { Grad }} & 1
\end{array}\right)
$$

in $H_{0}\left(\partial_{\eta}+\eta\right) \otimes \mathcal{H}_{0}$. Here $\overbrace{\text { Grad }}^{\circ}$ denotes the time-dependent extension of the closure of Grad restricted to smooth vector fields with compact support in the underlying open set $\Omega$ of $\mathbb{R}^{3}$ and - div denotes the time-dependent extension of its $L^{2}(\Omega)$-adjoint. Analogously, we could consider the Neumann boundary condition

$$
\left(\begin{array}{ccc}
\operatorname{trace} C^{-1} \operatorname{trace}^{*} & 0 & \operatorname{trace} C^{-1} \\
0 & \partial_{0} & -\overbrace{\mathrm{Div}}^{\circ} \\
\text { trace }^{*} & -C \text { Grad } & 1
\end{array}\right)
$$

Here $\overbrace{\operatorname{Div}}^{\circ}$ denotes the the time-dependent extension of the closure of div restricted to smooth vector fields with compact support in the underlying open set $\Omega$ of $\mathbb{R}^{3}$ and - Grad is re-used to denote the time-dependent extension of its $L^{2}(\Omega)$-adjoint.

In both cases the system is formally of the abstract form

$$
N_{\partial_{0}}(Y):=\left(\begin{array}{ccc}
\operatorname{trace} C^{-1} \operatorname{trace}^{*} & 0 & \operatorname{trace} C^{-1} \\
0 & \partial_{0} & -Y \\
\operatorname{trace}^{*} & Y^{*} & 1
\end{array}\right)
$$

where $Y^{*} \in\{-C \overparen{\text { Grad }},-C$ Grad $\}$ is the $H_{0}\left(\partial_{\eta}+\eta\right) \otimes C^{1 / 2}\left[L^{2}(\Omega)\right]$-adjoint of $Y \in$ $\{\overbrace{\text { Div }}^{\circ}$, Div $\}$. However, it is still not fully clear how the above formal matrix system is to be understood entry by entry. Clearly, $N_{\partial_{0}}(Y)$ with domain

$$
D=\left(H_{0}\left(\partial_{\eta}+\eta\right) \otimes L^{2}(\Omega)\right) \oplus\left(H_{1}\left(\partial_{\eta}+\eta\right) \otimes D\left(Y^{*}\right)\right) \oplus\left(H_{0}\left(\partial_{\eta}+\eta\right) \otimes D(Y)\right)
$$

yields a well-defined operator in $H_{0}\left(\partial_{\eta}+\eta\right) \otimes \mathcal{H}_{0}$ and seemingly it would make sense to 
consider the system

$$
N_{\partial_{0}}(Y)\left(\begin{array}{c}
p \\
v \\
T
\end{array}\right)=\left(\begin{array}{l}
0 \\
f \\
0
\end{array}\right)
$$

in its natural sense as a partial differential-algebraic equation (PDAE). Unfortunately, $N_{\partial_{0}}$ - in contrast to the compressible case - is not invertible in the sense that

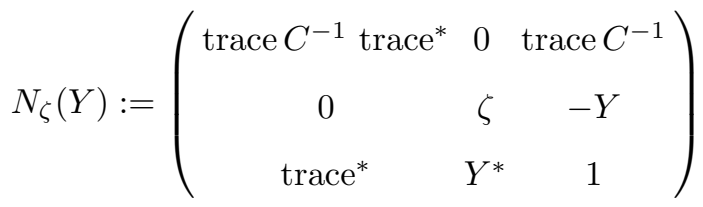

has in general no uniformly bounded inverse for $\zeta \in \mathrm{i}[\mathbb{R}]+\mathbb{R}_{\geq \eta}$, for some $\eta \in \mathbb{R}_{>0}$. Thus, our aim is to derive a well-posed substitute problem. This will be conveniently set in the framework of Sobolev lattices (extrapolation lattices) as developed in $[2,3]$.

1. Basic setup. We need a weaker concept of applying $Y$ and $Y^{*}$ as well as for timedifferentiation $\partial_{0}$. This will be accomplished by following construction, which we briefly recall from [2]. To construct the appropriate setting, let now $A: D(A) \subseteq H_{0} \rightarrow H_{1}$ denote an arbitrary densely defined, closed linear operator between Hilbert spaces $H_{0}, H_{1}$. We may now construct Sobolev lattices $\left(H_{k}(|A|+\mathrm{i})\right)_{k \in \mathbb{Z}}$ associated with the operator $(|A|+\mathrm{i})$. Here, $|A|=\sqrt{A^{*} A}$. We recall from [2] that the Hilbert spaces $H_{k}(|A|+\mathrm{i})$ are given as completions of $D\left(|A|^{k}\right)$ with respect to the norm

$$
u \mapsto|u|_{k, A}:=\left|(|A|+\mathrm{i})^{k} u\right|_{H_{0}},
$$

for $k \in \mathbb{Z}$. We also recall that $H_{k}(|A|+\mathrm{i})=\left(H_{-k}(|A|+\mathrm{i})\right)^{*}$ and that we have the continuous and dense embeddings $H_{v}(|A|+\mathrm{i}) \hookrightarrow H_{k}(|A|+\mathrm{i})$ for $v, k \in \mathbb{Z}, v \geq k$. We recall also that the mappings

$$
\begin{aligned}
D\left(|A|^{k+v}\right) \subseteq H_{v}(|A|+\mathrm{i}) & \rightarrow H_{v-k}(|A|+\mathrm{i}), \\
\Phi & \mapsto(|A|+\mathrm{i})^{k} \Phi,
\end{aligned}
$$

extend continuously to unitary mappings for $k, v \in \mathbb{Z}$ and we shall re-use the notation $(|A|+\mathrm{i})^{k}$ for these mappings. In the following we shall make use of this construction in particular for $A=Y: D(Y) \subseteq C^{1 / 2} L^{2}(\Omega) \rightarrow L^{2}(\Omega)$ and its adjoint $Y^{*}: D\left(Y^{*}\right) \subseteq$ $L^{2}(\Omega) \rightarrow C^{1 / 2} L^{2}(\Omega)$.

REMARK 1. Applying this construction likewise to the above skew-selfadjoint operator $\partial_{\eta}$ we obtain a chain of Hilbert spaces $\left(H_{k}\left(\left|\partial_{\eta}\right|+\mathrm{i}\right)\right)_{k \in \mathbb{Z}}$. Using $u \mapsto\left|\left(\partial_{\eta}+\eta\right)^{k} u\right|_{\eta, 0}$ as an equivalent norm we recover the spaces $H_{k}\left(\partial_{\eta}+\eta\right)$ introduced above, $k \in \mathbb{Z}$.

Denoting - as usual - the closure of the natural extension $\partial_{0}(\phi \otimes h):=\partial_{0} \phi \otimes h$, $A(\phi \otimes h):=\phi \otimes A h$ of $\partial_{0}, A$ to space- and time-dependent arguments by the same letters, we may now construct a Sobolev lattice $\left(H_{\eta, j, k, A}\right)_{j, k \in \mathbb{Z}}$ associated with the family 
$\left(\partial_{0},|A|\right)$, simply as tensor product spaces

$$
H_{\eta, j, k, A}:=H_{j}\left(\partial_{\eta}+\eta\right) \otimes H_{k}(|A|+\mathrm{i}) .
$$

We recall from [2] that the Hilbert spaces $H_{\eta, j, k, A}$ can be given as completions of the algebraic tensor product $\dot{C}_{\infty}(\mathbb{R}) \otimes D\left(|A|^{k}\right)$ with respect to the norm

$$
u \mapsto|u|_{\eta, j, k, A}:=\left|\partial_{0}^{j}(|A|+\mathrm{i})^{k} u\right|_{\eta, 0,0, A}:=\left|\partial_{0}^{j}(|A|+\mathrm{i})^{k} u\right|_{H_{0}\left(\partial_{\eta}+\eta\right) \otimes H_{0}},
$$

for $j, k \in \mathbb{Z}$. We also recall that

$$
H_{\eta, j, k, A}=\left(H_{\eta,-j,-k, A}\right)^{*}
$$

and that we have the continuous and dense embeddings $H_{\eta, u, v, A} \hookrightarrow H_{\eta, j, k, A}$ for $u, v, j, k \in$ $\mathbb{Z}, u \geq j, v \geq k$. The mappings

$$
\begin{aligned}
\check{C}_{\infty}(\mathbb{R}) \underset{a}{\otimes} D\left(|A|^{k+u}\right) \subseteq H_{\eta, u, v, A} & \rightarrow H_{\eta, u-j, v-k, A}, \\
\Phi & \mapsto \partial_{0}^{j}(|A|+\mathrm{i})^{k} \Phi,
\end{aligned}
$$

extend continuously to unitary mappings for $j, k, u, v \in \mathbb{Z}$ and we shall re-use again the notation $\partial_{0}^{j}(|A|+\mathrm{i})^{k}$ for these mappings. Note that there is no conflict with the laws of compositions of mappings, i.e. we have

$$
\partial_{0}^{j}(|A|+\mathrm{i})^{k} \partial_{0}^{u}(|A|+\mathrm{i})^{v}=\partial_{0}^{j+u}(|A|+\mathrm{i})^{k+v}
$$

for $j, k, u, v \in \mathbb{Z}$. The next question we need to answer is, how $A$ interacts with $|A|$. The answer is given by an abstract transmutation feature, which will be recorded in the following lemma.

Lemma. Let $A: D(A) \subseteq H_{0} \rightarrow H_{1}$ be a closed, densely defined linear operator between (complex) Hilbert spaces $H_{0}, H_{1}$. Then

$$
A|A|=\left|A^{*}\right| A
$$

in $H_{1}$ and on $D\left(|A|^{2}\right)=D(A|A|)=D\left(\left|A^{*}\right| A\right)$.

Proof. See corresponding lemmas in $[5,4]$.

We are now able to establish the meaning of the operator matrix equation

$$
\left(\begin{array}{ccc}
\operatorname{trace} C^{-1} \operatorname{trace}^{*} & 0 & \operatorname{trace} C^{-1} \\
0 & \partial_{0} & -Y \\
\text { trace }^{*} & Y^{*} & 1
\end{array}\right)\left(\begin{array}{l}
p \\
v \\
T
\end{array}\right)=\left(\begin{array}{l}
0 \\
f \\
0
\end{array}\right)
$$

in $H_{\eta, 0,0}:=H_{0}\left(\partial_{\eta}+\eta\right) \otimes \mathcal{H}_{0}=H_{\eta, 0,0, i d} \oplus H_{\eta, 0,0, Y^{*}} \oplus H_{\eta, 0,0, Y}$. Here $i d$ stands for the identity in the scalar $L^{2}(\Omega)$-space. Now, clearly the first equation

$$
\text { trace } C^{-1} \operatorname{trace}^{*} p+\operatorname{trace} C^{-1} T=0
$$

may be understood term by term in $H_{\eta, 0,0, i d}$, the second equation

$$
\partial_{0} v-Y T=f
$$

in $H_{\eta,-1,-1, Y^{*}}$ and the third equation

$$
Y^{*} v+T+\operatorname{trace}^{*} p=F
$$


simply in $H_{\eta, 0,0, Y}$. Thus, the entries of the operator matrix are meaningful and the proper domain of $N_{\partial_{0}}(Y)$ would be

$$
D\left(N_{\partial_{0}}(Y)\right):=\left\{\left(\begin{array}{c}
p \\
v \\
T
\end{array}\right) \in H_{\eta, 0,0} \mid\left(\begin{array}{ccc}
0 & 0 & 0 \\
0 & \partial_{0} & -Y \\
0 & Y^{*} & 0
\end{array}\right)\left(\begin{array}{c}
p \\
v \\
T
\end{array}\right) \in H_{\eta, 0,0}\right\} .
$$

2. The formulation of the abstract Stokes problem. We are now ready to explore the time-dependent formal initial boundary value problem for incompressible linear fluid flow as described by the Stokes problem. We start by considering the formal system of equations

$$
N_{\partial_{0}}(Y)\left(\begin{array}{c}
p \\
v \\
T
\end{array}\right)=\left(\begin{array}{l}
0 \\
f \\
0
\end{array}\right)
$$

Eliminating $T$ we obtain

$$
\begin{aligned}
\partial_{0} v+Y\left(Y^{*} v+\operatorname{trace}^{*} p\right) & =f \\
\operatorname{trace} C^{-1} Y^{*} v & =0 .
\end{aligned}
$$

Observing that

$$
\text { trace } C^{-1} Y^{*} \subseteq-\operatorname{div}
$$

and

$$
Y \text { trace* }^{*} \operatorname{grad}
$$

in the Dirichlet case and

$$
\operatorname{trace} C^{-1} Y^{*} \subseteq-\operatorname{div}
$$

and

$$
Y \text { trace }^{*} \subseteq \operatorname{grad}
$$

in the Neumann case, a more common if, however, slightly misleading form of the system can be obtained:

$$
\begin{aligned}
\operatorname{div} v & =0, \\
\partial_{0} v+Y Y^{*} v+\operatorname{grad} p & =f .
\end{aligned}
$$

Utilizing also that formally (only for $\mu, \kappa$ given by multiplication by constants!)

$$
-Y Y^{*} \subseteq \mu \Delta+(\mu+\kappa) \operatorname{grad} \operatorname{div}
$$

the system assumes the more familiar form

$$
\begin{aligned}
\operatorname{div} v & =0, \\
\partial_{0} v-\mu \Delta v-(\mu+\kappa) \operatorname{grad} \operatorname{div} v+\operatorname{grad} p & =f,
\end{aligned}
$$


in which, however, all the important information about operator domains has been lost. We shall therefore and for sake of generality prefer to use the above-given formulation of the problem.

If we eliminate $p$ (rather than $T$ ) to simplify the system, we obtain

$$
\left(\begin{array}{cc}
\partial_{0} & -Y \\
Y^{*} & \mathbb{E}
\end{array}\right)\left(\begin{array}{l}
v \\
T
\end{array}\right)=\left(\begin{array}{l}
f \\
0
\end{array}\right)
$$

with

$$
\mathbb{E}:=1-\operatorname{trace}^{*}\left(\operatorname{trace} C^{-1} \operatorname{trace}^{*}\right)^{-1} \operatorname{trace} C^{-1}
$$

as orthogonal projector onto the null space of trace $C^{-1}$. Indeed, we note that due to the scalar nature of (trace $C^{-1}$ trace*)

$$
\begin{aligned}
(1-\mathbb{E})^{2} & =\left(\operatorname{trace}{ }^{*}\left(\operatorname{trace} C^{-1} \operatorname{trace}^{*}\right)^{-1} \operatorname{trace} C^{-1}\right)^{2} \\
& =\left(\operatorname{trace} C^{-1} \operatorname{trace}^{*}\right)^{-2} \operatorname{trace}^{*}\left(\operatorname{trace} C^{-1} \operatorname{trace}^{*}\right) \operatorname{trace} C^{-1} \\
& =\left(\operatorname{trace} C^{-1} \operatorname{trace}^{*}\right)^{-1} \operatorname{trace}^{*} \operatorname{trace} C^{-1}
\end{aligned}
$$

and yields a selfadjoint operator. In this form the non-invertibility becomes apparent, since from

$$
\mathfrak{R e}\left\langle\left(\begin{array}{c}
v \\
T
\end{array}\right) \mid\left(\begin{array}{cc}
\partial_{0} & -Y \\
Y^{*} & \mathbb{E}
\end{array}\right)\left(\begin{array}{c}
v \\
T
\end{array}\right)\right\rangle_{\eta, 0,0} \geq \eta\langle v \mid v\rangle_{\eta, 0,0, Y^{*}}+\langle\mathbb{E} T \mid \mathbb{E} T\rangle_{\eta, 0,0, Y}
$$

we see that in general no boundedness information about the solution part $(1-\mathbb{E}) T$ is available. Recalling that

$$
\operatorname{trace} C^{-1} Y^{*} v=0
$$

we may, however, further reduce the system. We have for $\phi$ with $\operatorname{trace} C^{-1} Y^{*} \phi=0$

$$
\begin{aligned}
& \left\langle\phi \mid \partial_{0} v\right\rangle_{\eta, 0,0, Y^{*}}-\langle\phi \mid Y T\rangle_{\eta, 0,0, Y}=\langle\phi \mid f\rangle_{\eta, 0,0, Y^{*}}=\left\langle\phi \mid \partial_{0} v\right\rangle_{\eta, 0,0, Y^{*}}-\left\langle Y^{*} \phi \mid T\right\rangle_{\eta, 0,0, Y} \\
& =\left\langle\phi \mid \partial_{0} v\right\rangle_{\eta, 0,0, Y^{*}}-\left\langle\mathbb{E} Y^{*} \phi \mid T\right\rangle_{\eta, 0,0, Y}=\left\langle\phi \mid \partial_{0} v\right\rangle_{\eta, 0,0, Y^{*}}-\left\langle Y^{*} \phi \mid \mathbb{E} T\right\rangle_{\eta, 0,0, Y} \\
& =\left\langle\phi \mid \partial_{0} v\right\rangle_{\eta, 0,0, Y^{*}}+\left\langle Y^{*} \phi \mid Y^{*} v\right\rangle_{\eta, 0,0, Y}=\left\langle\phi \mid \partial_{0} v\right\rangle_{\eta, 0,0, Y^{*}}+\langle Z \phi \mid Z v\rangle_{\eta, 0,0, Z^{*}} \\
& =\langle\phi \mid P f\rangle_{\eta, 0,0, Y^{*}}
\end{aligned}
$$

with

$$
\begin{aligned}
Z: N\left(\operatorname{trace} C^{-1} Y^{*}\right) \subseteq \overline{N\left(\operatorname{trace} C^{-1} Y^{*}\right)} & \rightarrow\left\{T \in H_{\eta, 0,0, Y} \mid \operatorname{trace} C^{-1} T=0\right\} \\
v & \mapsto Y^{*} v,
\end{aligned}
$$

and where $P$ is an extension of the orthogonal projector onto $\overline{N\left(\operatorname{trace} C^{-1} Y^{*}\right)}$ as a closed subspace in $L^{2}(\Omega)$. To define $P$ properly we first note that for $f \in H_{\eta, 0,-1, Y^{*}}$ we have that $g_{f} \in H_{\eta, 0,-1, Z}$ given by

$$
\left\langle g_{f} \mid \phi\right\rangle_{0, Z}:=\langle f \mid \phi\rangle_{0}
$$


for all $\phi \in N\left(\operatorname{trace} C^{-1} Y^{*}\right)$ is uniquely determined. The mapping

$$
\begin{aligned}
P: H_{\eta, 0,-1, Y^{*}} & \rightarrow H_{\eta, 0,-1, Z}, \\
f & \mapsto g_{f},
\end{aligned}
$$

is therefore a well-defined continuous linear mapping. Indeed, we have

$$
|P f|_{\eta, 0,-1, Z}=\sup \left\{\left.\left|\langle f \mid \phi\rangle_{0}\right|\left|\phi \in N\left(\operatorname{trace} C^{-1} Y^{*}\right),\right| \phi\right|_{\eta, 0,1, Z} \leq 1\right\} \leq|f|_{\eta, 0,-1, Y^{*}}
$$

In view of the above calculation, we are led to consider

$$
S_{\partial_{0}}(Z):=\partial_{0}+Z^{*} Z
$$

in the Hilbert space $H_{0}\left(\partial_{\eta}+\eta\right) \otimes P\left[L^{2}(\Omega)\right]$. The operator $|Z|^{2}$ is is our representation of the Stokes operator, which is clearly a selfadjoint, nonnegative operator. We observe that the Stokes initial value problem

$$
\partial_{0} v+Z^{*} Z v=f_{0}+\delta \otimes v_{0}
$$

has a unique solution $v \in H_{\eta, 0,0, Z} \subseteq H_{\eta, 0,0, Y^{*}}$ for any given $f_{0} \in H_{\eta, 0,-1, Z}$ with $f_{0}=$ $\chi_{\mathbb{R}_{>0}}\left(m_{0}\right) f_{0}$ and $v_{0} \in H_{0, Z}$. This initial value problem would be a suitable well-posed replacement for the above Stokes problem. We shall take this as a solid starting point for our careful re-approach of the original question.

TheOREM. Let $f_{0} \in H_{\eta, 0,-1, Z}$ with $f_{0}=\chi_{\mathbb{R}_{\geq 0}}\left(m_{0}\right) f_{0}$ and $v_{0} \in H_{0, Z}$ be given. Then the Stokes initial value problem (24) has a unique solution $v \in H_{\eta, 0,1, Z}$ depending continuously on the data in the sense that

$$
\sqrt{\eta}|v|_{\eta, 0,0, Z}+|Z v|_{\eta, 0,0, Z^{*}} \leq 2 \frac{1}{\sqrt{\min \{1, \eta\}}}\left(\left|f_{0}\right|_{\eta, 0,-1, Z}+\sqrt{\pi}\left|v_{0}\right|_{0, Z}\right) .
$$

Proof. Since $\partial_{0}+|Z|^{2}=\partial_{0, \eta}+\eta+|Z|^{2}$ is boundedly invertible as a normal operator with $0 \in \varrho\left(\partial_{0, \eta}+\eta+|Z|^{2}\right)$, we obtain uniqueness. Indeed, for the difference $w \in H_{\eta, 0,1, Z}$ of two such solutions we have

$$
\left(\partial_{0, \eta}+\eta+|Z|^{2}\right) w=0
$$

holding in $H_{\eta,-1,-1, Z}$. Thus $\omega:=\left(\partial_{0, \eta}+\eta\right)^{-2}\left(|Z|^{2}+1\right)^{-1} w \in H_{\eta, 1,1, Z}$ also satisfies

$$
\left(\partial_{0, \eta}+\eta+|Z|^{2}\right) \omega=0
$$

implying $\omega=0$ and so also $w=0$.

As for the existence, we expect that

$$
v=\left(\partial_{0}+|Z|^{2}\right)^{-1} f_{0}+\left(\partial_{0}+|Z|^{2}\right)^{-1} \delta \otimes v_{0}
$$

is the solution we seek. We may consider the terms in the sum on the right hand side separately. Since $\partial_{0}+|Z|^{2}$ is indeed a normal operator with $\partial_{0, \eta}$ as imaginary part and $\eta+|Z|^{2}$ as real part, we obtain using the spectral theorem for $|Z|$ (with $\Pi_{|Z|}$ as associated 
spectral family)

$$
\begin{aligned}
& \left|\left(\partial_{0}+|Z|^{2}\right)^{-1} f_{0}\right|_{\eta, 0,0, Z} \\
& \leq\left|\left(\eta+|Z|^{2}\right)^{-1} f_{0}\right|_{\eta, 0,0, Z} \leq \sqrt{\int_{\mathbb{R}}\left|\left(\eta+|Z|^{2}\right)^{-1} f_{0}(t)\right|_{0, Z}^{2} \exp (-2 \eta t) d t} \\
& \leq \sqrt{\int_{\mathbb{R}} \int_{\mathbb{R}_{\geq 0}}\left(\left(1+\lambda^{2}\right)\left(\eta+\lambda^{2}\right)^{-2}\right)\left(1+\lambda^{2}\right)^{-1} d\left|\Pi_{|Z|}(\lambda) f_{0}(t)\right|_{0, Z}^{2} \exp (-2 \eta t) d t} \\
& \leq \frac{1}{\sqrt{\eta \min \{1, \eta\}}}\left|f_{0}\right|_{\eta, 0,-1, Z}
\end{aligned}
$$

and

$$
\begin{aligned}
& || Z\left|\left(\partial_{0}+|Z|^{2}\right)^{-1} f_{0}\right|_{\eta, 0,0, Z} \\
& \leq\left|(|Z|+\mathrm{i} \sqrt{\eta})\left(\eta+|Z|^{2}\right)^{-1} f_{0}\right|_{\eta, 0,0, Z} \leq\left|(\mathrm{i} \sqrt{\eta}-|Z|)^{-1} f_{0}\right|_{\eta, 0,0, Z} \\
& \leq \sqrt{\int_{\mathbb{R}}\left|\left(\mathrm{i} \sqrt{\eta}+|Z|^{2}\right)^{-1} f_{0}(t)\right|_{0, Z}^{2} \exp (-2 \eta t) d t} \\
& \leq \sqrt{\int_{\mathbb{R}} \int_{\mathbb{R}_{\geq 0}}\left(\left(1+\lambda^{2}\right)\left(\eta+\lambda^{2}\right)^{-1}\right)\left(1+\lambda^{2}\right)^{-1} d\left|\Pi_{|Z|}(\lambda) f_{0}(t)\right|_{0, Z}^{2} \exp (-2 \eta t) d t} \\
& \leq \frac{1}{\sqrt{\min \{1, \eta\}}}\left|f_{0}\right|_{\eta, 0,-1, Z} .
\end{aligned}
$$

For the second part we utilize the unitarity of the Fourier-Laplace transform with respect to time. We find

$$
\begin{aligned}
\| Z\left|\left(\partial_{0}+|Z|^{2}\right)^{-1} \delta \otimes v_{0}\right|_{\eta, 0,0, Z} & =|| Z\left|\left(\mathrm{i} m_{0}+\eta+|Z|^{2}\right)^{-1} v_{0}\right|_{0,0,0, Z} \\
& =\sqrt{\int_{\mathbb{R}}|| Z\left|\left(\mathrm{i} \tau+\eta+|Z|^{2}\right)^{-1} v_{0}\right|_{0, Z}^{2} d \tau} \\
& =\sqrt{\int_{\mathbb{R}} \int_{\mathbb{R}_{\geq 0}} \lambda^{2}\left|\mathrm{i} \tau+\eta+\lambda^{2}\right|^{-2} d\left|\Pi_{|Z|}(\lambda) v_{0}\right|_{0, Z}^{2} d \tau} \\
& =\sqrt{\int_{\mathbb{R}} \int_{\mathbb{R}_{\geq 0}} \lambda^{2}\left(\tau^{2}+\left(\eta+\lambda^{2}\right)^{2}\right)^{-1} d\left|\Pi_{|Z|}(\lambda) v_{0}\right|_{0, Z}^{2} d \tau} \\
& \leq \sqrt{\int_{\mathbb{R}_{\geq 0}} \int_{\mathbb{R}} \lambda^{2}\left(\tau^{2}+\left(\eta+\lambda^{2}\right)^{2}\right)^{-1}} d \tau d\left|\Pi_{|Z|}(\lambda) v_{0}\right|_{0, Z}^{2} \\
& \leq \sqrt{\int_{\mathbb{R}_{\geq 0}} \frac{\pi \lambda^{2}}{\left(\eta+\lambda^{2}\right)} d\left|\Pi_{|Z|}(\lambda) v_{0}\right|_{0, Z}^{2}} \\
& \leq \sqrt{\frac{\pi}{\min \{1, \eta\}}\left|v_{0}\right|_{0, Z}=\sqrt{\frac{\pi}{\min \{1, \eta\}}}\left|v_{0}\right|_{0, Y^{*}}}
\end{aligned}
$$


and similarly

$$
\begin{aligned}
\left|\left(\partial_{0}+|Z|^{2}\right)^{-1} \delta \otimes v_{0}\right|_{\eta, 0,0, Z} & =\left|\left(\mathrm{i} m_{0}+\eta+|Z|^{2}\right)^{-1} v_{0}\right|_{0,0,0, Z} \\
& =\sqrt{\int_{\mathbb{R}}\left|\left(\mathrm{i} \tau+\eta+|Z|^{2}\right)^{-1} v_{0}\right|_{0, Z}^{2} d \tau} \\
& =\sqrt{\int_{\mathbb{R}} \int_{\mathbb{R}_{\geq 0}}\left|\mathrm{i} \tau+\eta+\lambda^{2}\right|^{-2} d\left|\Pi_{|Z|}(\lambda) v_{0}\right|_{0, Z}^{2} d \tau} \\
& =\sqrt{\int_{\mathbb{R}} \int_{\mathbb{R}_{\geq 0}}\left(\tau^{2}+\left(\eta+\lambda^{2}\right)^{2}\right)^{-1} d\left|\Pi_{|Z|}(\lambda) v_{0}\right|_{0, Z}^{2}} d \tau \\
& \leq \sqrt{\int_{\mathbb{R}} \int_{\mathbb{R}_{\geq 0}}\left(\tau^{2}+\eta^{2}\right)^{-1} d\left|\Pi_{|Z|}(\lambda) v_{0}\right|_{0, Z}^{2} d \tau} \leq \sqrt{\frac{\pi}{\eta}}\left|v_{0}\right|_{0, Z} .
\end{aligned}
$$

These calculations show that $v:=\left(\partial_{0}+|Z|^{2}\right)^{-1} f_{0}+\left(\partial_{0}+|Z|^{2}\right)^{-1} \delta \otimes v_{0}$ is a well-defined solution in the stated class. Moreover, combining these estimates we find

$$
\sqrt{\eta}|v|_{\eta, 0,0, Z} \leq \frac{1}{\sqrt{\min \{1, \eta\}}}\left|f_{0}\right|_{\eta, 0,-1, Z}+\sqrt{\pi}\left|v_{0}\right|_{0, Z}
$$

and

$$
|Z v|_{\eta, 0,0, Z^{*}}=|| Z|v|_{\eta, 0,0, Z} \leq \frac{1}{\sqrt{\min \{1, \eta\}}}\left|f_{0}\right|_{\eta, 0,-1, Z}+\sqrt{\frac{\pi}{\min \{1, \eta\}}}\left|v_{0}\right|_{0, Z} .
$$

Adding these inequalities yields the desired continuity estimate.

From the solution theory we also have $v \in H_{\eta, 0,1, Z} \subseteq H_{\eta, 0,1, Y^{*}}$ and so $Y^{*} v \in$ $H_{\eta, 0,0, Z} \subseteq H_{\eta, 0,0, Y}$ as well as $Y Y^{*} v \in H_{\eta, 0,-1, Y^{*}}$. Consequently,

$$
\partial_{0} v+Y Y^{*} v-f-\delta \otimes v_{0}=: w \in H_{\eta,-1,-1, Y^{*}}
$$

If we take $\phi \in H_{\eta, 1,1, Y^{*}}$, then

$$
\begin{aligned}
\langle\phi \mid w\rangle_{\eta, 0,0, Y^{*}} & =\left\langle\phi \mid \partial_{0} v+Y Y^{*} v-f-\delta \otimes v_{0}\right\rangle_{\eta, 0,0, Y^{*}} \\
& =\left\langle\phi \mid \partial_{0} v\right\rangle_{\eta, 0,0, Y^{*}}+\left\langle Y^{*} \phi \mid Y^{*} v\right\rangle_{\eta, 0,0, Y}-\langle\phi \mid f\rangle_{\eta, 0,0, Y^{*}}-\left\langle\phi(0) \mid v_{0}\right\rangle_{0, Y^{*}} \\
& =\left\langle\phi \mid \partial_{0} v\right\rangle_{\eta, 0,0, Y^{*}}+\left\langle Y^{*} \phi \mid Z v\right\rangle_{\eta, 0,0, Y}-\langle\phi \mid f\rangle_{\eta, 0,0, Y^{*}}-\left\langle\phi(0) \mid v_{0}\right\rangle_{0, Y^{*}}
\end{aligned}
$$

and specializing to $\phi \in H_{1}\left(\partial_{\eta}+\eta\right) \otimes H_{1}(|Z|+\mathrm{i}) \subseteq H_{\eta, 1,1, Y^{*}}$ we obtain

$$
\begin{aligned}
\langle\phi \mid w\rangle_{\eta, 0,0, Y^{*}} & =\left\langle\phi \mid \partial_{0} v\right\rangle_{\eta, 0,0, Y^{*}}+\langle Z \phi \mid Z v\rangle_{\eta, 0,0, Z^{*}}-\langle\phi \mid P f\rangle_{\eta, 0,0, Y^{*}}-\left\langle\phi(0) \mid v_{0}\right\rangle_{0, Y^{*}} \\
& =\left\langle\phi \mid \partial_{0} v+Z^{*} Z v-P f-\delta \otimes v_{0}\right\rangle_{\eta, 0,0, Z}=0
\end{aligned}
$$

for all $\phi \in H_{1}\left(\partial_{\eta}+\eta\right) \otimes H_{1}(|Z|+\mathrm{i})=H_{1}\left(\partial_{\eta}+\eta\right) \otimes N\left(\operatorname{trace} C^{-1} Y^{*}\right)$. Thus, $w \in H_{\eta,-1,-1, Y^{*}}$ is in the so-called annihilator $\left(H_{1}\left(\partial_{\eta}+\eta\right) \otimes N\left(\operatorname{trace} C^{-1} Y^{*}\right)\right)^{a}$ of the subspace $H_{1}\left(\partial_{\eta}+\eta\right)$ $\otimes N\left(\operatorname{trace} C^{-1} Y^{*}\right) \subseteq H_{\eta, 1,1, Y^{*}}$.

Thus we finally have arrived at the proper reformulation of the problem originally posed, which we therefore refer to as the abstract Stokes problem

$$
\partial_{0} v+Y Y^{*} v-f-\delta \otimes v_{0} \in\left(H_{1}\left(\partial_{\eta}+\eta\right) \otimes N\left(\operatorname{trace} C^{-1} Y^{*}\right)\right)^{a} .
$$


3. The solution theory of the abstract Stokes problem. In summary of our previous calculations we find the existence part of the following theorem giving the desired "soft analysis" solution theory for the abstract Stokes problem.

THEOREM. Let $f \in H_{\eta, 0,-1, Y^{*}}$ such that $f=\chi_{\mathbb{R}_{\geq 0}}\left(m_{0}\right) f$ and $v_{0} \in N\left(\operatorname{trace} C^{-1} Y^{*}\right)$ be given, $\eta \in \mathbb{R}_{>0}$. Then, there is a unique solution $v \in H_{\eta, 0,1, Y^{*}}$ of relation (25). This solution depends continuously on the data in the sense that

$$
\sqrt{\eta}|v|_{\eta, 0,0, Y^{*}}+\left|Y^{*} v\right|_{\eta, 0,0, Y} \leq 2 \frac{1}{\sqrt{\min \{1, \eta\}}}\left(|f|_{\eta, 0,-1, Y^{*}}+\sqrt{\pi}\left|v_{0}\right|_{0, Y^{*}}\right) .
$$

Proof. For the existence part we follow the above argument and let $v \in H_{\eta, 0,1, Z} \subseteq$ $H_{\eta, 0,1, Y^{*}}$ be the unique solution of (24) with $f_{0}=P f$ as ensured by theorem 2 . We have derived that $v$ is then also a solution of (25), which proves existence.

Uniqueness follows, since a solution of (25) with $f=0$ and $v_{0}=0$ satisfies

$$
\left\langle\phi \mid \partial_{0} v+Y Y^{*} v\right\rangle_{\eta, 0,0, Y^{*}}=0
$$

for all $\phi \in H_{1}\left(\partial_{\eta}+\eta\right) \otimes N\left(\operatorname{trace} C^{-1} Y^{*}\right)$. Since

$0=\left\langle\phi \mid \partial_{0} v+Y Y^{*} v\right\rangle_{\eta, 0,0, Y^{*}}=\left\langle\phi \mid \partial_{0} v\right\rangle_{\eta, 0,0, Z}+\langle Z \phi \mid Z v\rangle_{\eta, 0,0, Z^{*}}=\left\langle\phi \mid \partial_{0} v+Z^{*} Z v\right\rangle_{\eta, 0,0, Z}$,

we have

$$
\partial_{0} v+Z^{*} Z v=0 .
$$

Thus, uniqueness is clear from the uniqueness for the Stokes evolutionary problem (24). It remains to derive the stated continuity estimate, which can be obtained by considering (24). By using that

$$
|v|_{\eta, 0,0, Y^{*}}=|v|_{\eta, 0,0, Z},|Z v|_{\eta, 0,0, Z^{*}}=\left|Y^{*} v\right|_{\eta, 0,0, Y},\left|v_{0}\right|_{0, Z}=\left|v_{0}\right|_{0, Y^{*}}
$$

and employing the continuity estimate $(22)$ for $P$ we obtain

$$
\sqrt{\eta}|v|_{\eta, 0,0, Y^{*}}+\left|Y^{*} v\right|_{\eta, 0,0, Y} \leq \frac{2}{\sqrt{\min \{1, \eta\}}}|f|_{\eta, 0,-1, Y^{*}}+\sqrt{\pi}\left(1+\frac{1}{\sqrt{\min \{1, \eta\}}}\right)\left|v_{0}\right|_{0, Y^{*}}
$$

as desired.

REMARK 2. In general, we have (note that trace* $\left[H_{\eta,-1,0, \text { id }}\right] \subseteq H_{\eta,-1,0, Y}$ )

$$
Y \operatorname{trace}^{*}\left[H_{\eta,-1,0, \text { id }}\right] \subseteq\left(H_{1}\left(\partial_{\eta}+\eta\right) \otimes N\left(\operatorname{trace} C^{-1} Y^{*}\right)\right)^{a} \subseteq H_{\eta,-1,-1, Y^{*}}
$$

but equality only holds under particular circumstances which, however, as we can see from the above theorem, are irrelevant for obtaining a solution theory for problem (25). If, however,

$$
Y \operatorname{trace}^{*}\left[H_{\eta,-1,0, \text { id }}\right]=\left(H_{1}\left(\partial_{\eta}+\eta\right) \otimes N\left(\operatorname{trace} C^{-1} Y^{*}\right)\right)^{a}
$$

happens to hold then we have

$$
w=Y \operatorname{trace}^{*} p
$$

for some $p \in H_{\eta,-1,0, \text { id }}$ and letting

$$
T:=-Y^{*} v-\operatorname{trace}^{*} p \in H_{\eta,-1,0, Y}
$$


we can recover the familiar equations we heuristically started out with. Indeed, only if (26) holds we have

$$
\partial_{0} v+Y T=\partial_{0} v+Y Y^{*} v+Y \operatorname{trace}^{*} p=f+\delta \otimes v_{0}
$$

holding in $H_{\eta,-1,-1, Y^{*}}$ for some $p \in H_{\eta,-1,0, \text { id }}$ and

$$
\text { trace } C^{-1} Y^{*} v=0
$$

holding in $H_{\eta, 0,0, Y}$. Condition (26) characterizes when the abstract Stokes system actually yields a solution of what one could call the classical Stokes system (28), (27), (29).

\section{References}

[1] R. Leis, Initial Boundary Value Problems in Mathematical Physics, Wiley and Teubner, 1986.

[2] R. Picard, Evolution equations as operator equations in lattices of Hilbert spaces, Glas. Mat. III Ser. 35 (2000), 111-136.

[3] R. H. Picard, Evolution equations as space-time operator equations, J. Math. Anal. Appl. 173 (1993), 436-458.

[4] R. Picard, Linear thermo-elasticity in nonsmooth media, Math. Methods Appl. Sci., 28 (2005), 2183-2199.

[5] R. Picard, On a model of electromagnetic field propagation in ferro-electric media, J. Math. Anal. Appl. 328 (2007), 655-675.

[6] H. Sohr, The Navier-Stokes Equations. An Elementary Functional Analytic Approach, Birkhäuser, Basel, 2001.

[7] W. Varnhorn, The Stokes Equations, Math. Res. 76, Akademie Verlag, Berlin, 1994. 